\title{
37 Evolution fattens its progeny
}

For millions of years, our evolution has been characterized by limited nutritional resources. Nutritional deficiency was therefore constantly much more dangerous than nutritional surplus and optimal utilization of food always provided a survival benefit.

From this perspective, the XXXL people of today belong to the evolutionary elite. A claim to fame most of them would be glad to divorce themselves from. Sixty percent of Germans are overweight. As many as one-eighth of all children and adolescents are too fat. The tragic consequence of this fact is that most overweight 10- to 13-year-olds will also be too heavy as adults. Among others, they are at an increased risk for high blood pressure, developing coronary heart disease, stroke, bowel cancer and diabetes already in middle age (Bjorge et al. 2008, Adams et al. 2014, Bairdain et al. 2014). Increased body size in childhood is associated with a greater risk of diabetes in adulthood, as con-

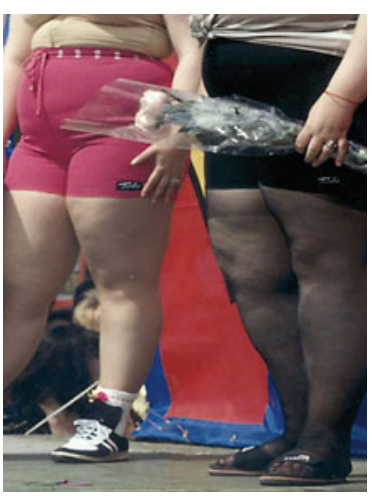

- Fig. 37.1 Source: dpa/akg firmed by analyses of the data on over 109,000 participants in Nurses' Health Study II. However, the risk normalizes when the affected women become lean again later in young adulthood (Yeung et al. 2010).

There are genetic co-factors respon- sible for overweight and obesity. They not only influence the distribution of adipose tissue ( $\triangleright$ Chapter 38), but are also involved in the regulation of feelings of hunger and satiation (Speliotes et al. 2010). However, the effects of genes overall is rather low, the risk for an elevated body mass index is mainly determined (to approximately $95 \%$ ) by a person's lifestyle. For hundreds and hundreds of generations, our genetic potential has stayed the same while the prevalence of obesity has just about doubled compared to 30 years ago (Finucane et al. 2011).

"Glutton: one who digs his grave with his teeth." (French proverb).

A too-high energy intake leading to weight gain may sometimes be unintentional. In these rather rare cases, the human intestinal flora is responsible for this. Depending on their composition, the approximately 50-100 trillion microorganisms living there in symbiosis with our body are capable of recovering energy media - more or less effectively - from the indigestible waste products of our metabolism, e.g. glucose from cellulose. This happens frequently when there is an imbalance in the proportions of bacteria colonizing the intestine, with more inhabitants from the phylum Firmicutes and a decreased number from the phylum Bacteroides. Dietary modifications can change the intestinal flora rapidly, even within as little as 24 hours (David et al. 2014). 